\title{
Preparation and evaluation of a set of bis(methoxycarbonylmethylthio) heteroquinones as CDC25B phosphatase inhibitors
}

\author{
Tatiana Besseta, Emmanuelle Braud ${ }^{b}, *$, Rafika Jarrayb, Christiane Garbayb, Stéphanie Kolbb, \\ Pierre-Marc Léo a and Christophe Morina,* \\ a Département de Chimie Moléculaire (CNRS, UMR 5250, ICMG FR-2607), Université Joseph Fourier, 301 Rue de la chimie, F-38402 Grenoble Cedex, France \\ ${ }^{\mathrm{b}}$ Laboratoire de Chimie et Biochimie Pharmacologiques et Toxicologiques, CNRS UMR 8601, Université Paris Descartes, 45 Rue des Saints Pères, F-75006 Paris, \\ France \\ *Corresponding author at: Laboratoire de Chimie et Biochimie Pharmacologiques et Toxicologiques, CNRS UMR 8601, Université Paris Descartes, \\ 45 Rue des Saints Pères, F-75006 Paris, France. Tel.: +33.142864085; fax: +33.142864082. E-mail address: emmanuelle.braud@parisdescartes.fr (E. Braud), \\ christophe.morin@ujf-grenoble.fr (C.Morin).
}

ARTICLE INFORMATION
Received: 03 February 2011
Received in revised form: 25 March 2011
Accepted: 29 March 2011
Online: 31 December 2011
KEYWORDS
Heterocyclic quinones
CDC25B inhibitors
Enzymatic activity
Phosphatase
Cancer
Cell cycle

\begin{abstract}
A set of new heteroquinone derivatives bearing two methoxycarbonylmethylthio groups on the benzoquinone ring were synthesized and evaluated for CDC25B phosphatase inhibitory activity. All compounds inhibited the enzyme with $\mathrm{IC}_{50}$ values in the micromolar range regardless of the size and heteroatoms constituting the heterocycle fused to the quinone ring. Moreover, these quinonoid-based compounds showed moderate antiproliferative activity toward two cancer cell lines (HeLa and MiaPaca-2). These results provide additional data for CDC25 inhibition by quinone-type derivatives and highlight the importance of substituents on the quinonic moiety.
\end{abstract}

\section{Introduction}

Cell signaling is regulated by covalent and reversible phosphorylation and dephosphorylation reactions, which are controlled by protein kinases and phosphatases. These signaling pathways are responsible for essential cell events such as growth, division or death, their deregulation leading to the apparition of pathologies such as cancers. Cell components involved in cell cycle regulation and in the checkpoint machinery are considered as promising targets in therapeutic and especially for cancer treatment. Indeed, inhibiting cell cycle progression will limit cell proliferation. Among possible targets, cyclin-dependent kinases (CDK) are currently widely studied. Among CDK regulators, CDC25 phosphatases have received increasing interest over the past years. These enzymes play a central role in cell cycle control in eukaryotes and in the checkpoint response to DNA damage [1-3]. Up-regulation of CDC25 has been observed in a wide variety of human cancers $[4,5]$ and the phosphatases are also involved in oncogenic transformation [6].

Consequently, many efforts have been devoted to search for CDC25 inhibitors. The most potent are currently quinonoidbased structures, which have been extensively studied [7]. Compounds $\mathbf{1}$ and $\mathbf{2}$ are lead compounds with $\mathrm{IC}_{50}$ values of 5 $\mu \mathrm{M}$ and $125 \mathrm{nM}$, respectively (Figure 1) [8,9]. They led to the preparation of a number of quinonic derivatives including heteroquinones [10] based on quinoline [11-17], quinazoline [11], phtalazine [11], quinoxaline [16], isoquinoline $[11,12,18]$, isoindole [19], pyrazole [19], isoxazole [19], isothiazole [19] and imidazole $[14,19]$ scaffolds. Since sub-micromolar activities and encouraging selectivities were observed with benzothiazolequinone, 11, and benzoxazolequinone, 12, [19] (Figure 1), these two pharmacophores were assembled into a bis-quinone moiety, which resulted in a ca. 10-fold increase in CDC25 phosphatase inhibition. In addition, this compound was demonstrated to inhibit the growth of human tumor xenografted on mice [20].

We previously reported the synthesis and the inhibitory activity of naphthoquinones and quinolinequinones, 3-6, bearing two identical alkylthio substituents on the quinone ring, these inhibitors displaying $\mathrm{IC}_{50}$ values in the micromolar range (Figure 1) [17,21]. Searching for a potent pharmacophore for CDC25 inhibition, Lazo and colleagues identified the bis-substituted naphthoquinone, $2,\left(\mathrm{IC}_{50}=125\right.$ $\mathrm{nM}$ ) as well as quinoleine, isoquinoleine and imidazole derivatives, 7-10, as micromolar CDC25 inhibitors [12].

In order to evaluate the effects of five-membered heterocycles fused to the quinone ring, Lavergne et al. synthesized and evaluated a set of heterocyclic quinones [19]. They observed that the nature of the heterocycle could modulate the activity of the quinonic derivatives monosubstituted with a $N, N$-dimethylethylenediamino group on the quinone ring. In this study, the most potent inhibitors $\mathbf{1 1}$ and 12 displayed IC $_{50}$ values in the hundred nanomolar range [19].

In order to investigate the influence, on CDC25 phosphatase activity, of five and six-membered heterocycles fused to the 1,4-dione pharmacophore substituted with two alkylthio groups, we designed, synthesized and evaluated a set of nine heteroquinones bearing two methoxycarbonyl methylthio substituents on the quinone core. 
<smiles>CC1=C(SCCO)C(=O)c2ccccc2C1=O</smiles>

1<smiles>[R]CSC1=C(SC[R])C(=O)c2ncccc2C1=O</smiles>

5: $\mathrm{R}=\mathrm{CO}_{2} \mathrm{H}$ 6: $\mathrm{R}=\mathrm{CH}_{2} \mathrm{CO}_{2} \mathrm{H}$

10<smiles>O=C1C(SCCO)=C(SCCO)C(=O)c2ccccc21</smiles>

2<smiles>[R]OC(=O)CSC1=C(SCC(=O)O[R])C(=O)c2ccccc2C1=O</smiles>

3: $\mathbf{R}=\mathbf{H}$

4: $\mathrm{R}=\mathrm{CH}_{2} \mathrm{C}_{6} \mathrm{H}_{5}$<smiles>[R]CSC1=C(SC[R])C(=O)c2[Y]([Y])cccc2C1=O</smiles>

7a: $\mathrm{X}=\mathrm{CH}, \mathrm{Y}=\mathrm{N}, \mathrm{R}=\mathrm{CH}_{2}$-O-THP 8a: $X=C H, Y=N, R=C_{2}-\mathrm{OC}(=0)-P h$

7b: $\mathrm{X}=\mathrm{N}, \mathrm{Y}=\mathrm{CH}, \mathrm{R}=\mathrm{CH}_{2}$-O-THP 9a: $X=C H, Y=N, R=$

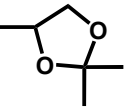
8b: $X=\mathrm{N}, \mathrm{Y}=\mathrm{CH}, \mathrm{R}=\mathrm{CH}_{2}-\mathrm{OC}(=\mathrm{O})-\mathrm{Ph}$ 9b: $X=N, Y=C H, R=$ $\int_{0}^{0}$<smiles>Cc1nc2c(s1)C(=O)C=C(NCCN(C)C)C2=O</smiles>

11

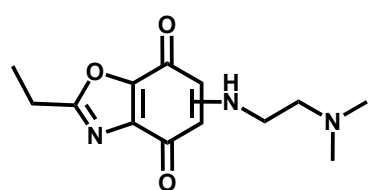

12

Figure 1. Inhibitors of CDC25 phosphatases.

\section{Experimental}

\subsection{Instrumentation}

Melting points were determined on a Büchi B-545 apparatus and are uncorrected. ${ }^{1} \mathrm{H}$ and ${ }^{13} \mathrm{C}$ NMR spectra were measured on a Bruker Avance 300 or Avance III 400 spectrometers. IR spectra were recorded on a Nicolet Magna IR-550 FT-IR spectrometer using $\mathrm{KBr}$ pellets or an ATR module. Mass spectra were recorded on a Bruker Daltonics Esquire $3000+$ or a Thermo Fischer Scientific Polaris Q ion-trap spectrometer. High resolution mass spectra were obtained using a Thermoquest Orbitrap apparatus. Elemental analyses (Service d'Analyse du Département de Chimie Moléculaire de Grenoble, France) were performed using a CHN analyzer built by the Service Central d'Analyses du CNRS (Vernaison, France).

\subsection{Synthesis}

\subsubsection{Preparation of compounds 14-17 and 21-26}

\subsubsection{2,3-Bis(1-methoxycarbonylmethylthio)-1,4- naphthoquinone (14)}

To a mixture of 2,3-dichloro-1,4-naphthoquinone $(50 \mathrm{mg}$, $0.22 \mathrm{mmol}$ ) and pyridine (50 $\mu \mathrm{L}, 0.62 \mathrm{mmol})$ in ethanol $(1 \mathrm{~mL})$ at $0{ }^{\circ} \mathrm{C}$ methyl thioglycolate $(0.484 \mathrm{mmol}, 43 \mu \mathrm{L})$ was added. The mixture was stirred at $4{ }^{\circ} \mathrm{C}$ for 68 hours then diluted with ethanol. After filtration and evaporation of the volatiles, the residue was recrystallized from hot acetone to give pure $\mathbf{1 4}$ as dark violet needles. Yield: $43 \%$. M.p.: $113^{\circ} \mathrm{C}$. FT-IR (film): 2939, $1738 \vee(\mathrm{C}=0), 1650 \vee(\mathrm{C}=0), 1583 \vee($ Aromatic $\mathrm{C}=\mathrm{C}), 1435,1276$, 1164, $1140 \mathrm{~cm}^{-1} .{ }^{1} \mathrm{H}$ NMR $\left(400 \mathrm{MHz}, \mathrm{CDCl}_{3}, \delta, \mathrm{ppm}\right): 8.03(\mathrm{~m}$, $2 \mathrm{H}, \mathrm{Ar}-\mathrm{H}), 7.68(\mathrm{~m}, 2 \mathrm{H}, \mathrm{Ar}-\mathrm{H}), 4.02\left(\mathrm{~s}, 4 \mathrm{H}, 2 \times \mathrm{CH}_{2}\right), 3.70(\mathrm{~s}, 6 \mathrm{H}$, $\left.2 \times \mathrm{CH}_{3}\right) .{ }^{13} \mathrm{C} \mathrm{NMR}\left(100 \mathrm{MHz}, \mathrm{CDCl}_{3}, \delta, \mathrm{ppm}\right): 178.8(2 \mathrm{C}=0), 169.3$ (2C=0), 146.2 (2C), 133.7 (2C), 132.8 (2C), 127.0 (2C), 52.7 (2C), $35.5(2 \mathrm{C})$. MS $\left(\mathrm{ESI}^{+}, \mathrm{m} / \mathrm{z}\right): 367\left[(\mathrm{M}+\mathrm{H})^{+}\right], 389\left[(\mathrm{M}+\mathrm{Na})^{+}\right]$.
Anal. calcd. for $\mathrm{C}_{16} \mathrm{H}_{14} \mathrm{O}_{6} \mathrm{~S}_{2}$ : C, 52.45; H, 3.86. Found: C, 52.71; H, 4.23 .

2.2.1.2. 6,7-Bis(1-methoxycarbonylmethylthio)-5,8quinolinequinone (15)

Under argon, to a solution of methyl thioglycolate $(98 \mu \mathrm{L}$, $0.97 \mathrm{mmol})$ in THF $(1 \mathrm{~mL})$ was added triethylamine $(135 \mu \mathrm{L}$, $0.97 \mathrm{mmol}$ ) and after $15 \mathrm{~min}$ 6,7-dichloroquinoline-5,8-dione $(100 \mathrm{mg}, 0.44 \mathrm{mmol})$. After $1 \mathrm{~h}$ stirring, diethyl ether $(5 \mathrm{~mL})$ was added, which was followed by filtration. The residue obtained after evaporation of the volatiles was purified by column chromatography on silica gel (ethyl acetate) to afford 15 as a red oil. Yield: $51 \% .{ }^{1} \mathrm{H}$ NMR $\left(300 \mathrm{MHz}, \mathrm{CDCl}_{3}, \delta, \mathrm{ppm}\right)$ : 8.94 (dd, $1 \mathrm{H}, J=4.5,1.5 \mathrm{~Hz}, \mathrm{Ar}-\mathrm{H}), 8.34$ (dd, $1 \mathrm{H}, J=7.8,1.5 \mathrm{~Hz}$, Ar-H), 7.62 (dd, 1H, J = 7.8, 4.5 Hz, Ar-H), 4.08 (s, $2 \mathrm{H}, \mathrm{CH}_{2}$ ), 4.00 $\left(\mathrm{s}, 2 \mathrm{H}, \mathrm{CH}_{2}\right), 3.68\left(\mathrm{~s}, 3 \mathrm{H}, \mathrm{CH}_{3}\right), 3.67\left(\mathrm{~s}, 3 \mathrm{H}, \mathrm{CH}_{3}\right) .{ }^{13} \mathrm{C}$ NMR $(75$ $\left.\mathrm{MHz}, \mathrm{CDCl}_{3}, \delta, \mathrm{ppm}\right): 177.9$ (C=0), $177.2(\mathrm{C}=0), 169.0(\mathrm{C}=0)$, 168.9 (C=0), 154.3 (C), 148.2 (C), 147.8 (C), 144.4 (C), 134.8 (C), 129.7 (C), 127.4 (C), 52.7 (C), 52.6 (C), 35.3 (C), 35.2 (C). MS $\left(\mathrm{ESI}^{+}, \mathrm{m} / \mathrm{z}\right): 368\left[(\mathrm{M}+\mathrm{H})^{+}\right], 390\left[(\mathrm{M}+\mathrm{Na})^{+}\right]$. HRMS $\left(\mathrm{ESI}^{+}\right)$ calcd. for $\mathrm{C}_{15} \mathrm{H}_{14} \mathrm{NO}_{6} \mathrm{~S}_{2}\left[(\mathrm{M}+\mathrm{H})^{+}\right]$: 368.02571. Found: 368.02591.

\subsubsection{6,7-Bis(1-methoxycarbonylmethylthio)-5,8- phthalazinequinone (16)}

A solution of methyl thioglycolate $(26 \mu \mathrm{L}, 0.28 \mathrm{mmol})$ and triethylamine ( $40 \mu \mathrm{L}, 0.28 \mathrm{mmol})$ in THF $(0.7 \mathrm{~mL})$ was stirred at room temperature during $15 \mathrm{~min}$ before addition of 6,7dichloro-5,8-phtalazinequinone ( $30 \mathrm{mg}, 0.13 \mathrm{mmol}$ ). After $2 \mathrm{~h}$ stirring, $\mathrm{CH}_{2} \mathrm{Cl}_{2}$ and water were added and the aqueous layer was extracted (x3) with $\mathrm{CH}_{2} \mathrm{Cl}_{2}$. The combined organic layers were washed with brine, dried over $\mathrm{Na}_{2} \mathrm{SO}_{4}$ and evaporated under reduced pressure to afford 6,7-bis(methoxycarbonyl methylthio)-5,8-dihydroxyphthalazine (37 $\mathrm{mg})$ as a brown oil $\left({ }^{1} \mathrm{H}\right.$ NMR $\left(400 \mathrm{MHz}, \mathrm{CDCl}_{3}, \delta, \mathrm{ppm}\right): 9.82$ (s, 2H, Ar-H), 3.67 (s, $4 \mathrm{H}, 2 \times \mathrm{CH}_{2}$ ), 3.65 (s, $6 \mathrm{H}, 2 \times \mathrm{CH}_{3}$ ). IR (film, $v, \mathrm{~cm}^{-1}$ ): 3383, 2953, 
2924, 1737, 1435, 1291, 1197, 1162, 1127, 1009. MS (ESI+, $\left.\mathrm{m} / \mathrm{z}): 371\left[(\mathrm{M}+\mathrm{H})^{+}\right]\right)$which was oxidized without purification. Thus $11 \mathrm{mg}(0.030 \mathrm{mmol})$ of it was stirred in acetonitrile:water (1:1) $(200 \mu \mathrm{L})$ to which was added dropwise a solution of 41 $\mathrm{mg}$ of cerium ammonium nitrate $(0.075 \mathrm{mmol})$ in acetonitrile:water $(20: 1)(320 \mu \mathrm{L})$. The mixture was then stirred at room temperature for $30 \mathrm{~min}$, before being diluted with an ice-water slurry $(5 \mathrm{~mL})$ and $\mathrm{CH}_{2} \mathrm{Cl}_{2}(5 \mathrm{~mL})$. After separation of the layers, the aqueous phase was extracted with $\mathrm{CH}_{2} \mathrm{Cl}_{2}(3 \times 5 \mathrm{~mL})$, the organic layers were combined and washed with brine $(5 \mathrm{~mL})$, dried over $\mathrm{Na}_{2} \mathrm{SO}_{4}$ and evaporated under reduced pressure. Preparative TLC $\left(\mathrm{Et}_{2} \mathrm{O}\right)$ gave $\mathbf{1 6}$ as a red oil. Yield: 10\%. FT-IR (film, cm-1): 3054, 2986, 2955, 2925, 1738 $v(\mathrm{C}=0), 1666 v(\mathrm{C}=0), 1461,1438,1265,1158,896 .{ }^{1} \mathrm{H}$ NMR ( $\left.400 \mathrm{MHz}, \mathrm{CDCl}_{3}, \delta, \mathrm{ppm}\right): 9.75$ (s, 2H, Ar-H) 4.08 (s, 4H, $\left.2 \times \mathrm{CH}_{2}\right), 3.72\left(\mathrm{~s}, 6 \mathrm{H}, 2 \times \mathrm{CH}_{3}\right) .{ }^{13} \mathrm{C} \mathrm{NMR}\left(100 \mathrm{MHz}, \mathrm{CDCl}_{3}, \delta, \mathrm{ppm}\right)$ : $177.5(2 \mathrm{C}=0), 168.9$ (2C=0), $146.3(2 \mathrm{C}), 146.1(2 \mathrm{C}), 125.4(2 \mathrm{C})$, $52.9(2 \mathrm{C}), 35.1(2 \mathrm{C}) . \mathrm{MS}\left(\mathrm{ESI}^{+}, \mathrm{m} / z\right): 369\left[(\mathrm{M}+\mathrm{H})^{+}\right]$. HRMS $\left(\mathrm{ESI}^{+}\right)$ calcd. for $\mathrm{C}_{14} \mathrm{H}_{13} \mathrm{~N}_{2} \mathrm{O}_{6} \mathrm{~S}_{2}(\mathrm{M}+\mathrm{H})+: 369.02095$, found: 369.02098 .

\subsubsection{2-Methyl-5,6-bis(1-methoxycarbonylmethylthio)-4,7- benzoxazolequinone (17)}

To a solution of $\mathbf{2 1}(80 \mathrm{mg}, 0.34 \mathrm{mmol})$ in ethanol $(1.7 \mathrm{~mL})$ was added pyridine $(80 \mu \mathrm{L}, 0.99 \mathrm{mmol})$ and the reaction mixture was cooled to $0{ }^{\circ} \mathrm{C}$ before methyl thioglycolate $(70 \mu \mathrm{L}$, $0.76 \mathrm{mmol}$ ) was added. Then, the reaction mixture was stirred at room temperature for $18 \mathrm{~h}$ before being diluted with $\mathrm{CH}_{2} \mathrm{Cl}_{2}$ $(5 \mathrm{~mL})$ and water $(5 \mathrm{~mL})$ and the aqueous layer was extracted with $\mathrm{CH}_{2} \mathrm{Cl}_{2}(3 \times 5 \mathrm{~mL})$. The organic layers were combined, washed with brine, dried over $\mathrm{Na}_{2} \mathrm{SO}_{4}$ and evaporated under reduced pressure to afford a burgundy-red oil $(25 \mathrm{mg})$ which is a mixture of $\mathbf{1 7}$ and 5,6-bis(methoxycarbonylmethylthio)-4,7dihydroxy-2-methyl-benzo[d]oxazole $\left({ }^{1} \mathrm{H}\right.$ NMR $(400 \mathrm{MHz}$, $\mathrm{CDCl}_{3}, \delta, \mathrm{ppm}$ ): 8.62 (br s, $\left.2 \mathrm{H}, \mathrm{OH}\right), 4.05\left(\mathrm{~s}, 4 \mathrm{H}, 2 \times \mathrm{CH}_{2}\right.$ ), 3.73 (s, $\left.\left.6 \mathrm{H}, 2 \times \mathrm{CH}_{3}\right), 2.66\left(\mathrm{~s}, 3 \mathrm{H}, \mathrm{CH}_{3}\right)\right)$. This mixture was diluted with $\mathrm{CHCl}_{3}: \mathrm{EtOH}(1: 1)(220 \mu \mathrm{L})$ and cooled to $0{ }^{\circ} \mathrm{C}$ before the addition of a solution of $\mathrm{FeCl}_{3} .6 \mathrm{H}_{2} \mathrm{O}(122 \mathrm{mg}, 0.45 \mathrm{mmol})$ in water $(110 \mu \mathrm{L})$. After stirring for $30 \mathrm{~min}$ at room temperature, $\mathrm{CH}_{2} \mathrm{Cl}_{2}(5 \mathrm{~mL})$ and water $(5 \mathrm{~mL})$ were added. The aqueous layer was extracted with $\mathrm{CH}_{2} \mathrm{Cl}_{2}(3 \times 5 \mathrm{~mL})$ and the combined organic layers were washed with brine $(5 \mathrm{~mL})$, dried over $\mathrm{Na}_{2} \mathrm{SO}_{4}$ and the volatiles were removed under reduced pressure. The dark purple oil thus obtained was purified by column chromatography on silica gel (Et 20 :pentane, 1:1) to afford 17 as a dark red oil. Yield: $79 \%$. FT-IR $\left(\mathrm{KBr}, \mathrm{cm}^{-1}\right)$ : 3054, 2987, $1739 v(\mathrm{C}=0), 1678 v(\mathrm{C}=0), 1421,1265,896 .{ }^{1} \mathrm{H}$ NMR $(400$ $\left.\mathrm{MHz}, \mathrm{CDCl}_{3}, \delta, \mathrm{ppm}\right): 4.05\left(\mathrm{~s}, 2 \mathrm{H}, \mathrm{CH}_{2}\right), 3.99\left(\mathrm{~s}, 2 \mathrm{H}, \mathrm{CH}_{2}\right), 3.73$ (s, $\left.3 \mathrm{H}, \mathrm{CH}_{3}\right), 3.72\left(\mathrm{~s}, 3 \mathrm{H}, \mathrm{CH}_{3}\right), 2.55\left(\mathrm{~s}, 3 \mathrm{H}, \mathrm{CH}_{3}\right) .{ }^{13} \mathrm{C} \mathrm{NMR}(100 \mathrm{MHz}$, $\left.\mathrm{CDCl}_{3}, \delta, \mathrm{ppm}\right): 175.3(\mathrm{C}=0), 169.0(\mathrm{C}=0), 168.9(\mathrm{C}=0), 168.5$ $(\mathrm{C}=0), 163.8$ (C), 158.0 (C), 147.6 (C), 141.9 (C), 119.6 (C), 52.8 (C), 52.8 (C), 35.8 (C), 35.2 (C), 10.6 (C). MS (ESI $\left.{ }^{+}, m / z\right): 372$ $\left[(\mathrm{M}+\mathrm{H})^{+}\right], 394\left[(\mathrm{M}+\mathrm{Na})^{+}\right]$. HRMS $\left(\mathrm{ESI}^{+}\right)$calcd. for $\mathrm{C}_{14} \mathrm{H}_{14} \mathrm{NO}_{7} \mathrm{~S}_{2}$ $(\mathrm{M}+\mathrm{H})^{+}:$372.02062. Found: 372.02112 .

\subsubsection{5,6-Dichloro-2-methyl-4,7-benzo[d]oxazolequinone (21)}

Sodium chlorate (1.06 g, $10.0 \mathrm{mmol}$ ) was added in portions over a period of $30 \mathrm{~min}$ to a stirred solution of 4-hydroxy-2methylbenzoxazole (200 mg, $1.34 \mathrm{mmol})$ in conc. $\mathrm{HCl}(8 \mathrm{~mL})$ at $65^{\circ} \mathrm{C}$. After $3 \mathrm{~h}$ stirring at this temperature, the reaction mixture was cooled to room temperature before dilution with water $(20 \mathrm{~mL})$ and $\mathrm{CH}_{2} \mathrm{Cl}_{2}(20 \mathrm{~mL})$. The layers were separated and the aqueous phase was extracted with $\mathrm{CH}_{2} \mathrm{Cl}_{2}(3 \times 10 \mathrm{~mL})$. The organic layers were combined, washed with brine $(10 \mathrm{~mL})$, dried over $\mathrm{Na}_{2} \mathrm{SO}_{4}$ and evaporated under reduced pressure to afford an orange solid which was recrystallized from $\mathrm{CH}_{2} \mathrm{Cl}_{2} / \mathrm{MeOH}$ to give $\mathbf{2 1}$ as a yellow solid. Yield: 25\%. M.p.: 197-199 ${ }^{\circ} \mathrm{C}$. FT-IR $\left(\mathrm{KBr}, \mathrm{cm}^{-1}\right): 3387,1688$ v(C=0), 1616
$v(\mathrm{C}=0), 1543,1174,812 .{ }^{1} \mathrm{H}$ NMR $\left(400 \mathrm{MHz}, \mathrm{CD}_{3} \mathrm{OD}, \delta, \mathrm{ppm}\right)$ : $2.58\left(\mathrm{~s}, 3 \mathrm{H}, \mathrm{CH}_{3}\right) .{ }^{13} \mathrm{C}$ NMR $(100 \mathrm{MHz}$, Acetone, $\delta, \mathrm{ppm}): 173.4$ (C=0), 167.7 (C=0), 165.6 (C), 159.9 (C), 144.2 (C), 142.4 (C), $120.6(\mathrm{C}), 11.4(\mathrm{C})$. MS $\left(\mathrm{ESI}^{+}, \mathrm{m} / \mathrm{z}\right): 233\left[(\mathrm{M}+\mathrm{H})^{+}\right]$. Anal. calcd. for $\mathrm{C}_{8} \mathrm{H}_{3} \mathrm{Cl}_{2} \mathrm{NO}_{3}$ : C, 41.42; $\mathrm{H}, 1.31 ; \mathrm{N}, 6.04$. Found: $\mathrm{C}, 41.29 ; \mathrm{H}$, $1.54 ; \mathrm{N}, 5.67 \%$.

\subsubsection{5,6-Bis(1-methoxycarbonylmethylthio)-4,7-benzo [b]thiophenequinone (22)}

A solution of benzo[b]thiophene-4,7-quinone $(49 \mathrm{mg}, 0.30$ $\mathrm{mmol}$ ) in $95 \%$ EtOH ( $6 \mathrm{~mL}$ ) was added to a solution of methyl thioglycolate $(56 \mu \mathrm{L}, 0.62 \mathrm{mmol})$ in $95 \%$ EtOH $(6 \mathrm{~mL})$ The resulting solution was refluxed for $1 \mathrm{~h}$ and after cooling, it was diluted with water $(10 \mathrm{~mL})$ and $\mathrm{CH}_{2} \mathrm{Cl}_{2}(10 \mathrm{~mL})$. The layers were separated and the aqueous phase was extracted with $\mathrm{CH}_{2} \mathrm{Cl}_{2}(3 \times 10 \mathrm{~mL})$. The combined organic layers were washed with brine $(10 \mathrm{~mL})$, dried over $\mathrm{Na}_{2} \mathrm{SO}_{4}$ and evaporated under reduced pressure to afford light yellow oil $(70 \mathrm{mg})$. This oil was diluted with acetonitrile:water (1:1) $(1.4 \mathrm{~mL})$ before the dropwise addition of a solution of cerium ammonium nitrate (321 mg, $0.58 \mathrm{mmol}$ ) in 20:1 of acetonitrile:water $(2.7 \mathrm{~mL}$ ). The mixture was stirred at room temperature for $30 \mathrm{~min}$ before dilution with an ice/water slurry $(5 \mathrm{~mL})$ and $\mathrm{CH}_{2} \mathrm{Cl}_{2}(5 \mathrm{~mL})$. The layers were separated and the aqueous layer was extracted with $\mathrm{CH}_{2} \mathrm{Cl}_{2}(3 \times 5 \mathrm{~mL})$. The combined organic layers were washed with brine $(5 \mathrm{~mL})$, dried over $\mathrm{Na}_{2} \mathrm{SO}_{4}$ and evaporated under reduced pressure to afford a red solid (60 mg). This material was taken up in $95 \% \mathrm{EtOH}(4.5 \mathrm{~mL})$ and added to a solution of methyl thioglycolate ( $42 \mu \mathrm{L}, 0.47 \mathrm{mmol}$ ) in $95 \%$ EtOH $(4.5 \mathrm{~mL})$. The resulting solution was then refluxed for 140 min and after cooling, was diluted with water $(10 \mathrm{~mL})$ and $\mathrm{CH}_{2} \mathrm{Cl}_{2}(10 \mathrm{~mL})$. The layers were separated and the aqueous phase was extracted with $\mathrm{CH}_{2} \mathrm{Cl}_{2}(3 \times 10 \mathrm{~mL})$. The organic layers were combined, washed with brine $(10 \mathrm{~mL})$, dried over $\mathrm{Na}_{2} \mathrm{SO}_{4}$ and evaporated under reduced pressure to afford a brown oil (91 mg). Once this oil was taken up in acetonitrile:water (1:1) $(1.4 \mathrm{~mL})$ a solution of ceric ammonium nitrate $(333 \mathrm{mg}, 0.61$ $\mathrm{mmol})$ in acetonitrile:water $(20: 1)(2.7 \mathrm{~mL})$ was added dropwise. After stirring for 30 minutes, an ice/water slurry (5 $\mathrm{mL}$ ) and $\mathrm{CH}_{2} \mathrm{Cl}_{2}(5 \mathrm{~mL})$ were added. The aqueous layer was then extracted with $\mathrm{CH}_{2} \mathrm{Cl}_{2}(3 \times 5 \mathrm{~mL})$ and the combined organic layers were washed with brine $(5 \mathrm{~mL})$, dried over $\mathrm{Na}_{2} \mathrm{SO}_{4}$ and evaporated under reduced pressure to afford a dark purple oil that was purified by column chromatography $\left(\mathrm{CH}_{2} \mathrm{Cl}_{2}\right)$ over silica gel to give 22 as a purple oil. Yield: $40 \%$. FT-IR (film, $\left.\mathrm{cm}^{-1}\right)$ : 3054, 2987, 2955, $1736 v(\mathrm{C}=0), 1663 v(\mathrm{C}=0), 1437$, 1265, 1147. ${ }^{1} \mathrm{H}$ NMR $\left(300 \mathrm{MHz}, \mathrm{CDCl}_{3}, \delta, \mathrm{ppm}\right): 7.62(\mathrm{~d}, 1 \mathrm{H}, J=$ $4.8 \mathrm{~Hz}, \mathrm{Ar}-\mathrm{H}), 7.48(\mathrm{~d}, 1 \mathrm{H}, J=4.8 \mathrm{~Hz}, \mathrm{Ar}-\mathrm{H}), 4.00\left(\mathrm{~s}, 2 \mathrm{H}, \mathrm{CH}_{2}\right)$, $3.99\left(\mathrm{~s}, 2 \mathrm{H}, \mathrm{CH}_{2}\right), 3.71$ (br s, $\left.6 \mathrm{H}, 2 \times \mathrm{CH}_{3}\right) .{ }^{13} \mathrm{C}$ NMR $(100 \mathrm{MHz}$, $\left.\mathrm{CDCl}_{3}, \delta, \mathrm{ppm}\right): 174.9(\mathrm{C}=0), 173.5(\mathrm{C}=0), 169.2(\mathrm{C}=0), 169.1$ (C=0), 145.1 (C), 144.8 (C), 143.3 (C), 141.6 (C), 133.7 (C), 126.7 (C), 52.7 (C), 52.6 (C), 35.7 (C), 35.6 (C). MS (ESI+ $m / z)$ : $372\left[(\mathrm{M}+\mathrm{H})^{+}\right], 394 \quad\left[(\mathrm{M}+\mathrm{Na})^{+}\right]$. HRMS $\left(\mathrm{ESI}^{+}\right)$calcd. for $\mathrm{C}_{14} \mathrm{H}_{12} \mathrm{O}_{6} \mathrm{~S}_{3} \mathrm{Na}(\mathrm{M}+\mathrm{Na})^{+}:$394.96882. Found: 394.96943.

\subsubsection{2-Methyl-5,6-bis(1-methoxycarbonylmethylthio)-4,7-} benzo[d] thiazolequinone (23)

Compound 23 was obtained as a purple oil from 2-methyl4,7-benzo[d]thiazolequinone, using the procedure described for the preparation of 22. Yield: $46 \%$. FT-IR (film, $\mathrm{cm}^{-1}$ ): 3053 , 2986, $1739 v(\mathrm{C}=0), 1671 v(\mathrm{C}=0), 1422,1265 .{ }^{1} \mathrm{H}$ NMR $(400$ $\mathrm{MHz}, \mathrm{CDCl}_{3}, \delta, \mathrm{ppm}$ ): 4.04 (s, $2 \mathrm{H}, \mathrm{CH}_{2}$ ), 3.97 (s, $2 \mathrm{H}, \mathrm{CH}_{2}$ ), 3.71 (s, $\left.3 \mathrm{H}, \mathrm{CH}_{3}\right), 3.70\left(\mathrm{~s}, 3 \mathrm{H}, \mathrm{CH}_{3}\right), 2.81\left(\mathrm{~s}, 3 \mathrm{H}, \mathrm{CH}_{3}\right) .{ }^{13} \mathrm{C} \mathrm{NMR}(100 \mathrm{MHz}$, $\left.\mathrm{CDCl}_{3}, \delta, \mathrm{ppm}\right): 173.7(\mathrm{C}=0), 173.4(\mathrm{C}=0), 173.3(\mathrm{C}=0), 169.1$ (C), 169.0 (C), 153.2 (C), 145.3 (C), 143.5 (C), 140.0 (C), 52.8 (C), 52.7 (C), 35.7 (C), 35.6 (C), 20.0 (C). MS (ESI $\left.{ }^{+}, \mathrm{m} / \mathrm{z}\right): 410$ $\left[(\mathrm{M}+\mathrm{Na})^{+}\right]$. HRMS $\left(\mathrm{ESI}^{+}\right)$calcd. for $\mathrm{C}_{14} \mathrm{H}_{14} \mathrm{NO}_{6} \mathrm{~S}_{3}(\mathrm{M}+\mathrm{H})^{+}$: 387.99778, found: 387.99872 . 


\subsubsection{6,7-Bis(1-methoxycarbonylmethylthio)-5,8- quinazolinequinone (24)}

Compound 24 was obtained from 5,8-quinazolequinone using the procedure described for the preparation of $\mathbf{2 2}$. Yield: $31 \%$. FT-IR (film, cm $\left.{ }^{-1}\right)$ : 2996, 2954, $1737 v(\mathrm{C}=0), 1666$ $v(\mathrm{C}=0), 1566 v$ (Aromatic, C=C), 1435, 1280. ${ }^{1} \mathrm{H}$ NMR $(400 \mathrm{MHz}$, $\mathrm{CDCl}_{3}, \delta, \mathrm{ppm}$ ): 9.58 (s, 1H, Ar-H), $9.42(\mathrm{~s}, 1 \mathrm{H}, \mathrm{Ar}-\mathrm{H}), 4.09$ (s, 2H, $\left.\mathrm{CH}_{2}\right), 4.07\left(\mathrm{~s}, 2 \mathrm{H}, \mathrm{CH}_{2}\right), 3.70$ (s, $\left.3 \mathrm{H}, \mathrm{CH}_{3}\right) 3,68\left(\mathrm{~s}, 3 \mathrm{H}, \mathrm{CH}_{3}\right) .{ }^{13} \mathrm{C}$ NMR (100 MHz, $\left.\mathrm{CDCl}_{3}, \delta, \mathrm{ppm}\right): 176.8(\mathrm{C}=0), 176.4(\mathrm{C}=0), 168.9$ $(\mathrm{C}=0), 168.8(\mathrm{C}=0), 162.5(\mathrm{C}), 157.2(\mathrm{C}), 153.5(\mathrm{C}), 147.3(\mathrm{C})$, 145.5 (C), 125.3 (C), 52.9 (C), 52.8 (C), 35.2 (C), 35.1 (C). MS $\left(\mathrm{ESI}^{+}, \mathrm{m} / \mathrm{z}\right): 369\left[(\mathrm{M}+\mathrm{H})^{+}\right], 391\left[(\mathrm{M}+\mathrm{Na})^{+}\right]$. HRMS $\left(\mathrm{ESI}^{+}\right)$calcd. for $\mathrm{C}_{14} \mathrm{H}_{13} \mathrm{~N}_{2} \mathrm{O}_{6} \mathrm{~S}_{2}(\mathrm{M}+\mathrm{H})^{+}$: 369.02095 . Found: 369.02101 .

\subsubsection{6,7-Bis(1-methoxycarbonylmethylthio)-5,8- quinoxalinequinone (25)}

Methyl thioglycolate $(25 \mu \mathrm{L}, \quad 0.275 \quad \mathrm{mmol})$ and triethylamine ( $38 \mu \mathrm{L}, 0.275 \mathrm{mmol})$ were mixed in THF $(620 \mu \mathrm{L})$ at room temperature and stirred for $15 \mathrm{~min}$ before 5,8quinoxalinequinone $(20 \mathrm{mg}, 0.125 \mathrm{mmol})$ was added. After stirring for $12 \mathrm{~h}$, the volatiles were evaporated under reduced pressure and the residue was dissolved in water and then extracted with $\mathrm{CH}_{2} \mathrm{Cl}_{2}(3 \times 10 \mathrm{~mL})$. The combined organic layers were washed with brine $(15 \mathrm{~mL})$, dried over $\mathrm{Na}_{2} \mathrm{SO}_{4}$ and evaporated under reduced pressure to afford a yellow solid which was purified by column chromatography on silica gel (Et $20: \mathrm{MeOH}, 94: 6)$ and crystallized from hot ethanol to afford 5,8-dihydroxy-6,7-bis(1-methoxycarbonylmethylthio)quinoxaline (17 mg, $37 \%$ ) as a yellow solid. M.p.: $129-130{ }^{\circ} \mathrm{C} .{ }^{1} \mathrm{H}$ NMR (400 MHz, $\left.\mathrm{CDCl}_{3}, \delta, \mathrm{ppm}\right): 8.83(\mathrm{~s}, 2 \mathrm{H}, \mathrm{Ar}-\mathrm{H}), 8.01(\mathrm{~s}, 2 \mathrm{H}$, $\mathrm{OH}), 3.86\left(\mathrm{~s}, 4 \mathrm{H}, 2 \times \mathrm{CH}_{2}\right), 3.68\left(\mathrm{~s}, 6 \mathrm{H}, 2 \times \mathrm{CH}_{3}\right) .{ }^{13} \mathrm{C} \mathrm{NMR}(75 \mathrm{MHz}$, $\left.\mathrm{CDCl}_{3}, \delta, \mathrm{ppm}\right): 170.4(2 \mathrm{C}=0), 147.3$ (C), 144.3 (C), 133.6 (C), 119.3 (C), 52.7 (C), 36.7 (C). IR (KBr, v, $\left.\mathrm{cm}^{-1}\right): 1720,1439,1400$, $1278,1154,1121,1100$. HRMS $\left(\mathrm{ESI}^{+}\right)$calcd. for $\mathrm{C}_{14} \mathrm{H}_{14} \mathrm{~N}_{2} \mathrm{O}_{6} \mathrm{~S}_{2} \mathrm{Na}$ $(\mathrm{M}+\mathrm{Na})^{+}:$393.01855, Found: 393.01857. This material $(17 \mathrm{mg}$, $0.046 \mathrm{mmol})$ was taken-up in acetonitrile:water $(1: 1)(500 \mu \mathrm{L})$, to which was added dropwise under stirring a solution of cerium ammonium nitrate (55 mg, $0.101 \mathrm{mmol})$ in acetonitrile:water $(20: 1)(950 \mu \mathrm{L})$. The mixture was then stirred at room temperature for $30 \mathrm{~min}$ before being diluted with a ice/water slurry $(5 \mathrm{~mL})$ and $\mathrm{CH}_{2} \mathrm{Cl}_{2}(5 \mathrm{~mL})$. The aqueous phase was extracted with $\mathrm{CH}_{2} \mathrm{Cl}_{2}(4 \times 5 \mathrm{~mL})$ and the combined organic layers were washed with brine $(5 \mathrm{~mL})$, and dried over $\mathrm{Na}_{2} \mathrm{SO}_{4}$. Evaporation of the volatiles under reduced pressure afforded a dark purple oil which was purified by column chromatography on silica gel $\left(\mathrm{CH}_{2} \mathrm{Cl}_{2}\right.$ then $\left.\mathrm{CH}_{2} \mathrm{Cl}_{2}: \mathrm{MeOH}, 95: 5\right)$ to give 25 as an orange oil. Yield: 24\%. FT-IR (film, $\mathrm{cm}^{-1}$ ): 3003, 2954, 2924, 2851, $1731 v(\mathrm{C}=0), 1667 v(\mathrm{C}=0), 1530 v$ (Aromatic $\mathrm{C}=\mathrm{C}), 1485,1435,1286,1191,1004 .{ }^{1} \mathrm{H}$ NMR $\left(300 \mathrm{MHz}, \mathrm{CDCl}_{3}\right.$, $\delta, \mathrm{ppm}$ ): 8.69 (s, $2 \mathrm{H}, \mathrm{Ar}-\mathrm{H}), 4.12\left(\mathrm{~s}, 4 \mathrm{H}, 2 \times \mathrm{CH}_{2}\right), 3.70(\mathrm{~s}, 6 \mathrm{H}$, $\left.2 \times \mathrm{CH}_{3}\right) .{ }^{13} \mathrm{C}$ NMR $\left(75 \mathrm{MHz}, \mathrm{CDCl}_{3}, \delta, \mathrm{ppm}\right): 176.7(2 \mathrm{C}=0), 169.0$ (2C=0), 148.6 (C), 146.6 (C), 144.6 (C), 52.8 (C), 35.2 (C). MS $\left(\mathrm{ESI}^{+}, \mathrm{m} / \mathrm{z}\right): 369\left[(\mathrm{M}+\mathrm{H})^{+}\right], 391\left[(\mathrm{M}+\mathrm{Na})^{+}\right]$. Anal. calcd. for $\mathrm{C}_{14} \mathrm{H}_{12} \mathrm{~N}_{2} \mathrm{O}_{6} \mathrm{~S}_{2}$ : C, 45.65; H, 3.28; N, 7.60. Found: C, 45.89; H, 3.22; N, $7.23 \%$.

\subsubsection{5,6-Bis(1-methoxycarbonylmethylthio)-4,7- indolequinone (26)}

Methyl thioglycolate $(94 \mu \mathrm{L}, 1.05 \mathrm{mmol})$ and triethylamine $(150 \mu \mathrm{L}, 1.0 \mathrm{mmol})$ were added to THF $(4.8 \mathrm{~mL})$ and the mixture was stirred at room temperature for $15 \mathrm{~min}$ before the addition of 4,7-indolequinone (70 mg, $0.476 \mathrm{mmol}$ ). The reaction mixture was stirred for $16 \mathrm{~h}$, then was diluted with water $(5 \mathrm{~mL})$ and $\mathrm{CH}_{2} \mathrm{Cl}_{2}(5 \mathrm{~mL})$. The aqueous layer was extracted with $\mathrm{CH}_{2} \mathrm{Cl}_{2}(3 \times 10 \mathrm{~mL})$ and the combined organic layers were washed with brine $(15 \mathrm{~mL})$, dried over $\mathrm{Na}_{2} \mathrm{SO}_{4}$ and evaporated under reduced pressure. Purification of the crude product was achieved by column chromatography on silica gel $\left(\mathrm{CH}_{2} \mathrm{Cl}_{2}: \mathrm{AcOEt}, 94: 6\right)$ to give $\mathbf{2 6}$ as a purple oil. Yield: $36 \%$. FTIR $\left(\mathrm{KBr}, \mathrm{cm}^{-1}\right): 3300 v(\mathrm{~N}-\mathrm{H}), 2953,2926,1737 v(\mathrm{C}=0), 1649$ $v(\mathrm{C}=0), 1435,1381,1292,1194,1157,1124,1008 .{ }^{1} \mathrm{H}$ NMR (300 MHz, $\mathrm{CDCl}_{3}, \delta, \mathrm{ppm}$ ): 10.28 (br s, $\left.1 \mathrm{H}, \mathrm{NH}\right), 7.01$ (d, $1 \mathrm{H}$, $J=2.7 \mathrm{~Hz}, \mathrm{Ar}-\mathrm{H}$ ), 6.55 (d, $1 \mathrm{H}, J=2.7 \mathrm{~Hz}, \mathrm{Ar}-\mathrm{H}), 4.01\left(\mathrm{~s}, 2 \mathrm{H}, \mathrm{CH}_{2}\right)$, $3.94\left(\mathrm{~s}, 2 \mathrm{H}, \mathrm{CH}_{2}\right), 3.73\left(\mathrm{~s}, 3 \mathrm{H}, \mathrm{CH}_{3}\right), 3.72\left(\mathrm{~s}, 3 \mathrm{H}, \mathrm{CH}_{3}\right) .{ }^{13} \mathrm{C} \mathrm{NMR}$ $\left(75 \mathrm{MHz}, \mathrm{CDCl}_{3}, \delta, \mathrm{ppm}\right): 176.6(\mathrm{C}=0), 171.2(\mathrm{C}=0), 169.8$ $(\mathrm{C}=0), 169.6(\mathrm{C}=0), 146.8$ (C), 141.5 (C), 130.8 (C), 126.2 (C), 126.0 (C), 109.4 (C), 52.7 (C), 52.6 (C), 36.1 (C), 35.8 (C). MS $\left(\mathrm{ESI}^{+}, \mathrm{m} / \mathrm{z}\right): 356\left[(\mathrm{M}+\mathrm{H})^{+}\right], 378\left[(\mathrm{M}+\mathrm{Na})^{+}\right]$. HRMS $\left(\mathrm{ESI}^{+}\right)$calcd. for $\mathrm{C}_{14} \mathrm{H}_{14} \mathrm{NO}_{6} \mathrm{~S}_{2}(\mathrm{M}+\mathrm{H})^{+}$: 356.02571. Found: 356.02617. Anal. Calcd. for $\mathrm{C}_{14} \mathrm{H}_{13} \mathrm{NO}_{6} \mathrm{~S}_{2}$ : C, 47.31; H, 3.69; N, 3.94. Found: C, 47.89; $\mathrm{H}, 3.85 ; \mathrm{N}, 3.76 \%$.

\subsubsection{In vitro enzymatic assay}

The activity of the MBP-CDC25B [22] recombinant enzyme was monitored using fluorescein diphosphate. The assay was performed in 96-well plates in a final volume of $200 \mu \mathrm{L}$. MBPCDC25B3 was diluted in assay buffer [30 mM Tris- $\mathrm{HCl}(\mathrm{pH}=$ 8.2), $75 \mathrm{mM} \mathrm{NaCl}, 0.67 \mathrm{mM}$ EDTA, $0.033 \% \mathrm{BSA}, 1 \mathrm{mM}$ DTT] so that the final concentration was $90 \mathrm{ng} /$ well. The reaction was initiated by addition of $25 \mu \mathrm{M}$ of fluorescein diphosphate followed by immediate measurement of fluorescein phosphate emission with a Fluoroskan Ascent (Lab Systems; excitation filter: $485 \mathrm{~nm}$, emission filter: $530 \mathrm{~nm}$ ). For each compound, the drug concentration required for $50 \%$ inhibition $\left(\mathrm{IC}_{50}\right)$ was determined from a sigmoidal dose-response curve using GraphPad Prism 4 (GraphPad Software, San Diego, CA). Results are expressed as the mean of at least two independent experiments with three determinations per tested concentration and per experiment.

\subsubsection{Cell proliferation assay}

The inhibition of cell proliferation was determined using a colorimetric assay based on the cleavage of the WST-1 tetrazolium salt by mitochondrial dehydrogenases in viable cells, leading to formazan formation. At day 0, HeLa and MiaPaCa-2 cells were plated at 5500 and 5000 cells/well, respectively in 96-well culture plates with $95 \mu \mathrm{L}$ of medium/well. At day 1, cells were treated for $48 \mathrm{~h}$ with $5 \mu \mathrm{L}$ of increasing concentrations of drug (dissolved in DMSO, to a final concentration $<0.05 \%$ ). At day 3 , after addition of $10 \mu \mathrm{L}$ of WST- 1 per well, cells were incubated 2 hours at $37{ }^{\circ} \mathrm{C}$ in a humidified atmosphere of $5 \% \quad \mathrm{CO}_{2}$. Absorbance was then measured at $490 \mathrm{~nm}$ with a Bio-Rad microplate reader. The results are expressed as the mean of three independent experiments with three determinations per tested concentration and per experiment. The $\mathrm{IC}_{50}$ value were determined from a sigmoidal dose-response using GraphPad Prism (GraphPad Software, San Diego, CA).

\section{Results and discussion}

\subsection{Chemistry}

Parent compound bis(methoxycarbonylmethylthio) naphthaquinone, 14, was prepared from 6,7-dichloronaphthoquinone, 13, by nucleophilic attack of methyl mercaptoacetate [23].

Compounds 15 and 16 were obtained from 6,7-dichloro5,8-quinolinequinone, 18, [24] and 6,7-dichloro-5,8phthalazinequinone, 19, [24], respectively, using 'reverse addition' conditions (i.e. addition of the dichloroheteroquinone to a solution of sodium methoxy carbonylmethyl-thiolate). Derivative 17 was synthesized from 5,6-dichloro-2-methyl-4,7benzoxazolequinone, 21, prepared by oxidative halogenation of 7-hydroxy-2-methylbenzoxazole, 20, [25]. 
<smiles>[R7]CC(C)(C)OC(=O)CS</smiles>

13<smiles>COC(=O)CSC1=C(SCC(=O)OC)C(=O)c2ccccc2C1=O</smiles>

$14(43 \%)$

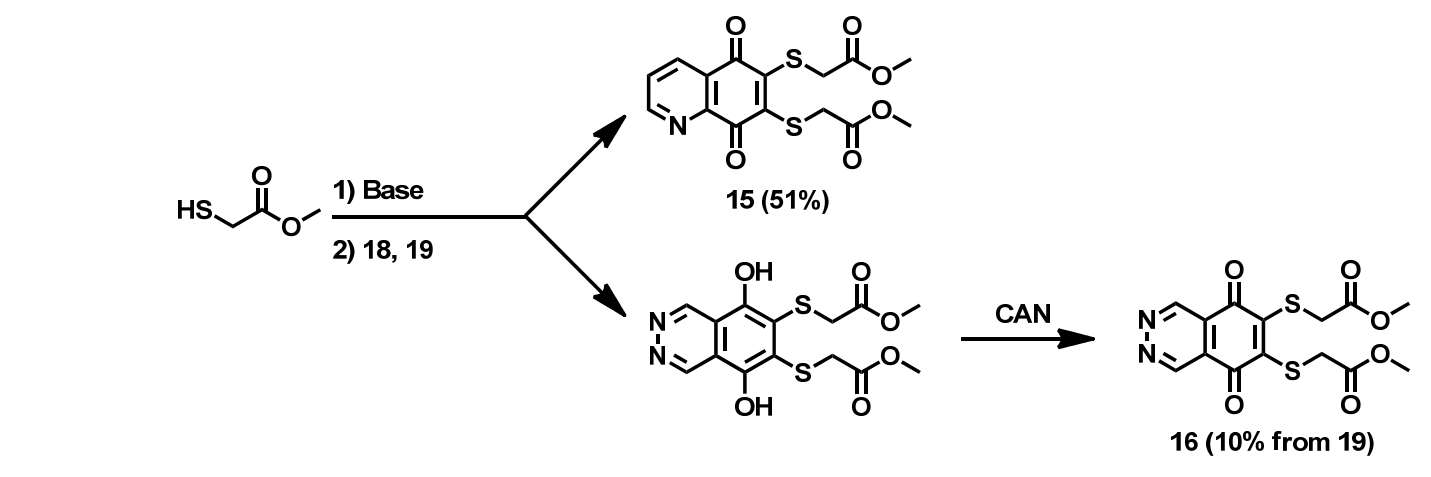

(1)

20

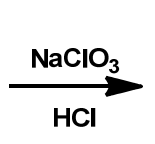<smiles>Cc1nc2c(o1)C(=O)C(Cl)=C(Cl)C2=O</smiles>

$21(25 \%)$

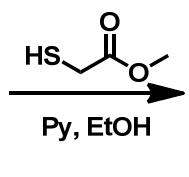

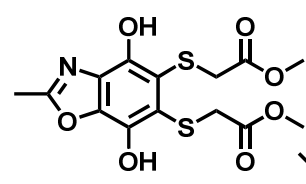

$\mathrm{FeCl}_{3}$<smiles>COC(=O)CSC1=C(SCC(=O)OC)C(=O)c2oc(C)nc2C1=O</smiles>

$17(79 \%$ from 21$)$

Scheme 1<smiles>CC1=C(C)C(=O)C=CC1=O</smiles><smiles>CCC</smiles><smiles>[R][R]1cc(O)c([12CH])c(O)c1</smiles><smiles>[R]C1=CC(=O)C([2H])=C([R])C1=O</smiles><smiles>[R]C1=C([R])C(=O)C([Hg])=C([Te])C1=O</smiles>

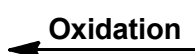

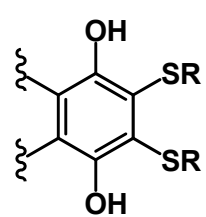

Figure 2. Addition of a thiol onto heteroquinones (regioisomers are not highlighted)

Since bis(methoxycarbonylmethyl-thio)hydroquinones are the primary products obtained from $\mathbf{1 9}$ or $\mathbf{2 1}$, an oxidation step was required which was carried out conventionally $\left(\mathrm{FeCl}_{3}\right.$ or CAN) (Scheme 1).

When thiols react with unsubstituted quinones, three intermediates are met en route to get a disubstituted quinone (Scheme 2). These can be observed spectroscopically but do not need to be isolated as a sequential addition/oxidation/ addition/oxidation process can be carried out up to the final product, as it has been previously exemplified for the preparation of bis(thio) derivatives of quinocarcin [26].

Accordingly, reacting benzo[b]thiophene-4,7-quinone $[27,28]$, 2-methyl-4,7-benzo[d] thiazolequinone [29-32] and 5,8-quinazolinequinone [33,34] with methyl mercaptoacetate, followed by oxidation (CAN) in a stepwise and duplicated manner (see experimental section), afforded bis-substituted heteroquinones, 22-24, (Figure 2). Concerning the 5,8quinoxalinequinone scaffold $[24,35,36]$, it yielded a bis(thio)hydroquinone in a single step which was followed by oxidation to get 25 . Moreover, in the case of indole-4,7-dione $[37,38]$, the bis-substituted quinone, 26, could be obtained directly, i.e. without the need for an external oxidizing agent, which presumably arises from relative redox properties of intermediate species within the reaction system.

Although bis(methoxycarbonylmethylthio)-heteroquinones, 14-17 and 22-26 have been isolated in fair-to-low (but unoptimized) yields (Figure 3), they have been obtained in pure state, which has allowed their evaluation on CDC25 phosphatase enzymatic activity. 
Table 1. Inhibitory activities of heteroquinones 14-17 and 22-26 toward CDC25.

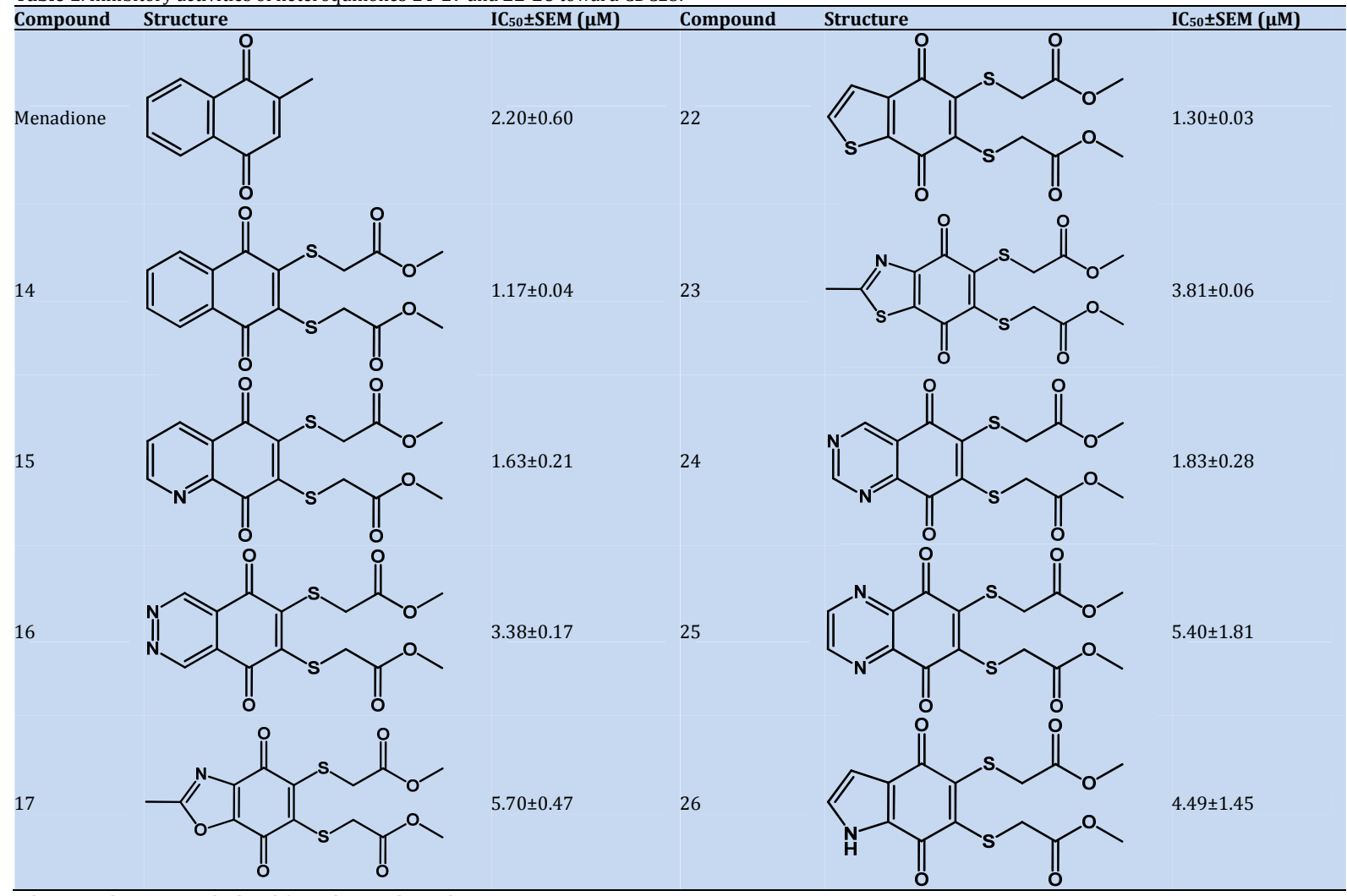

aThe IC 50 values were calculated from three independent experiments.<smiles>COC(=O)CSC1=C(SCC(=O)OC)C(=O)c2sccc2C1=O</smiles>

$22(40 \%)$<smiles>COC(=O)CSC1=C(SCC(=O)OC)C(=O)c2sc(C)nc2C1=O</smiles>

$23(46 \%)$<smiles>COC(=O)CSC1=C(SCC(=O)OC)C(=O)c2ncncc2C1=O</smiles>

$24(31 \%)$<smiles>COC(=O)CSC1=C(SCC(=O)OC)C(=O)c2nccnc2C1=O</smiles>

$25(9 \%)$<smiles>COC(=O)CSC1=C(SCC(=O)OC)C(=O)c2[nH]ccc2C1=O</smiles>

$26(36 \%)$

Figure 3. Structures of quinones, 22-26.

\subsection{Enzymatic activity studies}

The nine newly synthesized heteroquinones, 14-17 and 2226 were examined for their inhibitory activity against the recombinant protein MBP-CDC25B using fluorescein-3,6diphosphate (FDP) as the artificial substrate. The fluorescent emission resulting from dephosphorylation was monitored during $30 \mathrm{~min}$ at $30{ }^{\circ} \mathrm{C}$ in the presence of increasing concentrations of inhibitors [21]. Menadione was used as a reference. Results are reported in Table 1.

All tested quinones show comparable inhibitory activities with IC $\mathrm{C}_{50}$ values ranging from 1.17 to $5.70 \mu \mathrm{M}$, naphthoquinone being the most potent pharmacophore for this set of heteroquinones. With regard to the six-membered heterocyclic derivatives, 14-16, and 24-25, increasing the number of nitrogen atoms on the heterocycle led to a slight decrease of activity, quinoxalinequinone, 25, being the less potent inhibitor.

The parent compound $\mathbf{1 4}$ with two methyl ester groups is 10 -fold less potent than the bis(2-hydroxyethylthio) analogue NSC95397 [12] for which an IC 50 of $125 \mathrm{nM}$ was reported though we previously measured for this compound an $\mathrm{IC}_{50}$ value of $3.8 \mu \mathrm{M}$ under our conditions [21]. On the other hand, 14 displayed similar activity when compared with the biscarboxylic acid $\mathbf{3}$ and the corresponding benzyl di-ester $\mathbf{4}$ [21]. 
Table 2. In vitro growth inhibition of HeLa and MiaPaCa-2 cell lines.

\begin{tabular}{|c|c|c|c|c|}
\hline \multirow[t]{2}{*}{ Compound } & \multicolumn{2}{|c|}{ HeLa \% of growth inhibition } & \multicolumn{2}{|c|}{ MiaPaCa-2 \% of growth inhibition } \\
\hline & $100 \mu \mathrm{M}$ & $10 \mu \mathrm{M}$ & $100 \mu \mathrm{M}$ & $10 \mu \mathrm{M}$ \\
\hline Menadione & \multicolumn{2}{|c|}{$\mathrm{IC}_{50}=37.5 \pm 3.5 \mu \mathrm{M}$} & \multicolumn{2}{|l|}{$\mathrm{IC}_{50}=33 \mu \mathrm{M}$} \\
\hline 15 & $79 \pm 0.9$ & $13 \pm 0.9$ & $77 \pm 2.1$ & $24 \pm 15.4$ \\
\hline 16 & $60 \pm 2.1$ & $21.0 \pm 1.5$ & $74 \pm 0.7$ & $10 \pm 3.7$ \\
\hline 17 & $62 \pm 3.1$ & $6.0 \pm 4.1$ & $75 \pm 1.2$ & 0 \\
\hline 22 & \multicolumn{2}{|c|}{$\mathrm{IC}_{50}=26.9 \pm 6.3 \mu \mathrm{M}$} & \multicolumn{2}{|l|}{$\mathrm{IC}_{50}=29.0 \pm \mu \mathrm{M}$} \\
\hline 23 & \multicolumn{2}{|c|}{$\mathrm{IC}_{50}=34.5 \pm 4.9 \mu \mathrm{M}$} & $78 \pm 1.7$ & 27 \\
\hline 24 & $47.0 \pm 5.5$ & $22.0 \pm 3.0$ & $71 \pm 0.2$ & $4 \pm 3.6$ \\
\hline 25 & 0 & 0 & $24 \pm 4.6$ & $6 \pm 4.8$ \\
\hline 26 & $82.0 \pm 0.9$ & $8.0 \pm 6.2$ & $75 \pm 2.0$ & $5 \pm 5.0$ \\
\hline
\end{tabular}

In contrast, the bis-ester quinolinequinone $\mathbf{1 5}\left(\mathrm{IC}_{50}=1.63\right.$ $\mu \mathrm{M})$ is more than 5 -fold more potent than its bis-carboxylic acid counterpart ( $\mathrm{IC}_{50}=11.2 \mu \mathrm{M}$ for 5 ) [17].

Within the five-membered heterocycle sub-set, benzoxazolequinone, 17, and benzothiazolequinone, 23, bearing a methyl group on the heterocyclic moiety did not lead to the expected increase of activity when compared with $\mathbf{1 1}$ and $\mathbf{1 2}$ [19]. Extending the scope of the study to scaffolds such as benzothiophenequinone, 22, and indolequinone, 26, which had not been previously considered for CDC25 inhibition did not bring any improvement.

Finally, when comparing both sub-families, results indicate that the six-membered heterocyclic derivatives are slightly more active than the five-membered heterocyclic set, benzothiophenequinone, 22, being an exception.

\subsection{Antiproliferative activity}

The cytotoxicity of 8 quinonoid-based CDC25B inhibitors was evaluated on the human cancer cell lines HeLa (cervix) and MiaPaCa-2 (pancreatic adenocarcinoma) using a WST-1 colorimetric cleavage assay. The percentage of growth inhibition was determined at $100 \mu \mathrm{M}$ and $10 \mu \mathrm{M}$ for all compounds and the $\mathrm{IC}_{50}$ values were measured for the most efficient heterocyclic quinones only. Menadione was also tested and inhibited HeLa and MiaPaCa- 2 cell lines with comparable $\mathrm{IC}_{50}$ of 37.5 and $33 \mu \mathrm{M}$, respectively (Table 2 ).

All compounds showed cytotoxic activity toward both cell lines at $100 \mu \mathrm{M}$ with percentages of growth inhibition above $60 \%$ except derivative, 25, which was totally inactive on HeLa cells and displayed a percentage of $24 \%$ inhibition at $100 \mu \mathrm{M}$ toward the pancreatic cell line. These series of quinone is less potent on HeLa cells than compounds $\mathbf{1}$ and $\mathbf{2}$ for which we previously reported $\mathrm{IC}_{50}$ values of 8.8 and $10.8 \mu \mathrm{M}$, respectively [21]. Benzothiophenequinone, 22, which also showed one of the highest anti-phosphatase activity was the most cytotoxic quinone with activities comparable to that of menadione.

\section{Conclusion}

Quinonoid-based compounds are currently the most potent CDC25 phosphatase inhibitors. In the present study, we examined the effect on CDC25B activity of heterocycles fused to the 1,4-dione pharmacophore substituted with two thiol groups. For this limited series of bis-substituted heteroquinones, important modifications of the heterocyclic ring are well tolerated since all derivatives show similar micromolar activities. Unfortunately, we were not able to increase the affinity of the quinonic compounds by modulating the heterocyclic part as expected. These results underline the role of substitution of the quinone ring, which is essential for potent CDC25 inhibition [12,19]. Thus, for this set of quinonic derivatives, the substitution pattern appears to be responsible for the moderate inhibitory activity level and probably explains the absence of significant differences in activities between these heteroquinones. Therefore, it could be valuable to prepare and evaluate on $\mathrm{CDC} 25$ phosphatase activity heteroquinones bearing two $N, N$-dimethylethylenediamino or 2-hydroxyethylthio groups on the quinone ring.

\section{Acknowledgements}

Martine Fayolle is thanked for the preparation of 4hydroxy-2-methylbenzoxazole (20) and Tatiana Besset is grateful to the 'Ministère de l'Education Nationale, de la Recherche et de la Technologie' (MENRT) for a doctoral fellowship. MBP-CDC25B3 was provided by Bernard Ducommun.

\section{References}

[1]. Nilsson, I.; Hoffman, I. Prog. Cell Cycle Res. 2000, 4, 107-114.

[2]. Iliakis, G.; Wang, Y.; Guan, J.; Wang, H. Oncogene 2003, 22, 5834-5847.

[3]. Karlsson-Rosenthal, C.; Millar, J. B. Trends Cell Biol. 2006, 16, 285-292

[4]. Kristjansdottir, K.; Rudolph, J. Chem. Biol. 2004, 11, 1043-1051.

[5]. Boutros, R.; Dozier, C.; Ducommun B. Curr. Opin. Cell Biol. 2006, 18 185-191.

[6]. Galaktionov, K.; Chen, X.; Beach, D. Nature 1996, 382, 511-517.

[7]. Contour-Galcera, M. O.; Sidhu, A.; Prévost, G.; Bigg, D.; Ducommun, B. Pharmacol. Ther. 2007, 115, 1-12.

[8]. Nishikawa, Y.; Carr, B. I.; Wang, M.; Kar, S.; Finn, F.; Dowd, P.; Zheng, Z B.; Kerns, J.; Naganathan, S. J. Biol. Chem. 1995, 270, 28304-28310.

[9]. Tamura, K.; Southwick, E. C.; Kerns, J.; Rosi, K.; Carr, B. I.; Wilcox, C.; Lazo, J. S. Cancer Res. 2000, 60, 1317-1325.

[10]. Garuti, L.; Roberti, M.; Pizzirani, D. Curr. Med. Chem. 2008, 15, 573580.

[11]. Lazo, J. S.; Aslan, D. C.; Southwick, E. C.; Cooley, K. A.; Ducruet, A. P.; Joo, B.; Vogt, A.; Wipf P. J. Med. Chem. 2001, 44, 4042-4049.

[12]. Lazo, J. S.; Nemoto, K.; Pestell, K. E.; Cooley, K.; Southwick, E. C.; Mitchell, D. A.; Furey, W.; Gussio, R.; Zaharevitz, D. W.; Joo, B.; Wipf, P. Mol. Pharmacol. 2002, 61, 720-728.

[13]. Pu, L.; Amoscato, A. A.; Bier, M. E.; Lazo, J. S. J. Biol. Chem. 2002, 277 46877-46885.

[14]. Brisson, M.; Nguyen, T.; Wipf, P.; Joo, B.; Day, B. W.; Skoko, J. S. Schreiber, E. M.; Foster, C.; Bansal, P.; Lazo, J. S. Mol. Pharmacol. 2005, $68,1810-1820$.

[15]. Cossy, J.; Belotti, D.; Brisson, M.; Skoko, J. J.; Wipf, P.; Lazo, J. S. Bioorg. Med. Chem. 2006, 14, 6283-6287.

[16]. Keinan, S.; Paquette, W. D.; Skoko, J. J.; Beratan, D. N.; Yang, W.; Shinde, S.; Johnston, P. A.; Lazo, J. S.; Wipf, P. Org. Biomol. Chem. 2008, 6, 32563263.

[17]. Braud, E.; Goddard, M. L.; Kolb, S.; Brun, M. P.; Mondesert, O.; Quaranta, M.; Gresh, N.; Ducommun, B.; Garbay, C. Bioorg. Med. Chem. 2008, 16, 9040-9049.

[18]. Brisson, M.; Foster, C.; Wipf, P.; Joo, B.; Tomko Jr, R. J.; Nguyen, T. Lazo, J. S. Mol. Pharmacol. 2007, 71, 184-192.

[19]. Lavergne, O.; Fernandes, A. C.; Brehu, L.; Sidhu, A.; Brezak, M. C.; Prévost, G.; Ducommun, B.; Contour-Calcera, M. O. Bioorg. Med. Chem Lett. 2006, 16, 171-175.

[20]. Brezak, M. C.; Valette, A.; Quaranta, M.; Contour-Galcera, M. O.; Jullien, D.; Lavergne, O.; Frongia, C.; Bigg, D.; Kasprzyk, P. G.; Prevost, G. P.; Ducommun, B. Int. J. Cancer 2009, 124, 1449-1456.

[21]. Brun, M. P.; Braud, E.; Angotti, D.; Mondésert, 0.; Quaranta, M. Montes, M.; Miteva, M.; Gresh, N.; Ducommun, B.; Garbay, C. Bioorg Med. Chem. 2005, 13, 4871-4879. 
[22]. Brezak, M. C.; Quaranta, M.; Mondésert, O.; Galcera, M. O.; Lavergne, O.; Alby, F.; Cazales, M.; Baldin, V.; Thurieau, C.; Harnett, J.; Lanco, C.; Kasprzyk, P. G.; Prévost, G. P.; Ducommun, B. Cancer Res. 2004, 64, 3320-3325.

[23]. Shi, S.; Katz, T. J.; Yang, B. V.; Liu, L. J. Org. Chem. 1995, 60, 1285-1297.

[24]. Shaikh, I. A.; Johnson, F.; Grollman, A. P. J. Med. Chem. 1986, 29, $1329-$ 1340

[25]. Crabbe, P.; Villarino, A.; Muchowski, J. M. J. Chem. Soc. , Perkin Trans 1 1973, 2220-2222

[26]. Saito, H.; Hirata, T.; Kasai, M.; Fujimoto, K.; Ashizawa, T.; Morimoto, M.; Sato, A. J. Med. Chem. 1991, 34, 1959-1966.

[27]. Xu, J. Z.; Chen, C. J. Labelled Compd. Radiopharm. 2006, 49, 1187-1200.

[28]. Cherif, M.; Cotelle, P.; Catteau, J. P. Heterocycles 1992, 34, 1749-1758.

[29]. Could, S. J.; Shen, B.; Whittle, Y. G. J. Am. Chem. Soc. 1989, 111, 79327938

[30]. Lyon, M. A.; Lawrence, S.; Williams, D. J.; Jackson, Y. A. J. Chem. Soc. , Perkin Trans 1 1999, 437-442.

[31]. Fries, K.; Reitz, H. Liebigs Ann. 1937, 527, 38-60.

[32]. Kitahara, Y.; Nakahara, S.; Tanaka, Y.; Kubo, A. Heterocycles 1992, 34, 1623-1630.

[33]. Muchowski, J. M.; Maddox, M. L.; J. Mex. Chem. Soc. 2005, 49, 24-31.

[34]. Potts, K. T.; Bhattacharjee, D.; Walsh, E. B. J. Org. Chem. 1986, 51, 2011-2021.

[35]. Besset, T.; Morin, C. Synthesis 2009, 1753-1756.

[36]. Morin, C.; Besset, T.; Moutet, J. C.; Fayolle, M.; Brückner, M.; Limosin, D.; Becker, K.; Davioud-Charvet, E. Org. Biomol. Chem. 2008, 6, 27312742.

[37]. Showalter, H. D. H.; Pohlmann, G. Org. Prep. Proc. Int. 1992, 24, 484487.

[38]. Legentil, L.; Bastide, J.; Delfourne, E. Tetrahedron Lett. 2003, 44, 2473 2475. 